\title{
Effects of Hypophysectomy and Corticosterone Acetate Treatment on Hepatic Lipid Composition in the Chick (Gallus domesticus) Embryo
}

\author{
BYRoN A. DONEEN \\ Division of Biological Sciences, University of Michigan, Ann Arbor, Michigan 48109
}

Accepted May 16, 1980

\begin{abstract}
Lipid is transferred from yolk to liver and to other tissues at an accelerated rate after 16 days of incubation in the intact chicken embryo. Hypophysectomy (partial decapitation) at $36 \mathrm{hr}$ of incubation reduced liver lipid content on Days 16 and 18. The greatest losses on Day 18 were among cholesterol esters but decreased unesterified cholesterol and phospholipid were also observed. Treatment of hypophysectomized embryos with corticosterone acetate $(300 \mu \mathrm{g}$ per day on Days $13,14,15)$ partially restored total hepatic lipid content to control values on Day 16, with an even greater effect on 18 days of incubation. Most of the increase in lipid occurred in the triglyceride fraction. Liver cholesterol content also increased in response to hormone but neither cholesterol esters nor phospholipids were elevated by corticosterone acetate. Hypophysectomy interferes with the absorption of lipids from yolk and with fat deposition in embryonic liver. However, treatment of hypophysectomized embryos with corticosterone acetate did not correct the major defects in hepatic lipid content. Therefore, fat metabolism and transport of yolk lipids to the embryo during the last week of incubation does not depend exclusively on adrenal glucocorticoids.
\end{abstract}

Lipid metabolism in the avian embryo differs markedly from that observed in birds after hatching. This difference stems from the large amount of lipid stored in the yolk. Some yolk lipid components can be readily utilized by the embryo with only modest transformations in fatty acid composition (Noble and Moore, 1964). Lipogenesis, therefore, can be low in embryonic liver without loss of substrate required for lipid metabolism or for biosynthesis of cellular membranes (Goodridge, 1968a,b; Donaldson et al., 1971; Joshi and Sidbury, 1975; Freeman, 1978). The embryonic liver, however, can remodel fatty acid composition of phospholipids derived from yolk (Noble and Moore, 1967). Similar to reduced lipogenesis, lipolytic pathways which degrade triglycerides to fatty acids in liver and in adipose tissue are also poorly developed in the embryo (Goodridge, $1968 \mathrm{c}$; Langslow, 1972). Though $\beta$-oxidative pathways are active in the embryonic liver, fatty acid substrate can be obtained directly from yolk (Pugh and Sid- bury, 1971). Lipid is transferred from yolk to embryo by the yolk sac membrane. The rate of transport increases after 14 days of incubation and exceeds $1 \mathrm{~g} /$ day in the final 2 days of incubation in the chick (Noble and Moore, 1964).

In contrast to our better understanding of the endocrine regulation of lipid metabolism in the postnatal or adult bird (Fisher and Goodridge, 1978; Goodridge and Ball, 1967; Goodridge, 1978; Joshi and Aranda, 1979a, b; Langslow et al., 1979), hormones that regulate embryonic lipid metabolism or its major controlling step, the transport of lipid from yolk, have not yet been identified. A few experiments suggest important regulatory roles for some pituitary hormones and for adrenal glucocorticoids in embryonic lipid metabolism or transport. Hypophysectomy (partial decapitation) reduced hepatic lipid content after 16 days of incubation (Sandra and Thommes, 1977). Liver lipid content was partially restored in hypophysectomized embryos receiving pituitary trans- 
plants (Sandra and Thommes, 1977). Partial decapitation of chick embryos likewise depressed plasma cholesterol early in development (prior to 16 days), and this deficiency was corrected in part by a single treatment with cortisone acetate (Thommes and Shulman, 1967).

The purposes of this paper are first, to quantitatively characterize the ontogeny of some major hepatic lipid classes which are derived from yolk and deposited in the liver of the embryonic chick; and sccond, to observe the effects of hypophysectomy and corticosterone acetate treatment on liver lipid content and composition during the last week of incubation. These studies can decide if pituitary hormones and adrenal glucocorticoids are required for transfer of particular yolk lipids to the embryonic liver.

\section{METHODS}

Embryos. Fertilized eggs (Rhode Island Reds $x$ White Leghorn hybrids) were obtained locally from a commercial supplier. To begin the timed incubation period, eggs were transferred from $12^{\circ}$ to a humidified $(60 \%)$ stationary incubator $\left(37.5 \pm 0.5^{\circ}\right)$. Livers were examined in intact embryos on alternate days from Day 12 to Day 20. Livers were also examined in unfed chickens $12-24 \mathrm{hr}$ after hatching (Day 21). Hypophysectomized embryos were produced by partial decapitation according to Fugo (1940) between 36 and $40 \mathrm{hr}$ of incubation and eggs resealed with transparent tape. In some eggs the shell was opened and resealed but the embryo was not operated upon (windowed controls).

Hormone treatment. Corticosterone 21-acetate (Sigma) was dissolved in a small volume of $95 \%$ ethanol and then finely suspended in sterile $0.9 \%$ saline (final ethanol concentration, 2\%). Treated embryos received $300 \mu \mathrm{g}$ hormone each day on Days 13 , 14 , and 15 of incubation by application of $50 \mu \mathrm{l}$ hormone suspension to chlorioallantoic membrane. Vehicle-treated embryos received $50 \mu \mathrm{l} 2 \%$ ethanol in $0.9 \%$ saline.

Lipid isolation, separation, and measurement. Livers were dissected from embryos, weighed within 1 min, and frozen in dry ice-ethanol. Livers from 12day embryos were pooled in groups of two. Measurements on older embryos used individual livers. Livers were homogenized in chloroform:methanol (2:1) and lipid extracted according to Folch et al. (1957). Resuspended homogenate $(10 \mu l)$ was taken for measurement of protein using the method of Lowry et al. (1951) with bovine serum albumin as standard. The residue which remained after lipid extraction was dried and weighed. Residue weight was added to lipid weight determined later to yield the total dry weight. Lipid extracts were collected and solvent evaporated in pretared tubes. Lipid (1.0 to $1.5 \mathrm{mg}$ ) was applied in $2:$ chloroform:methanol to Whatman LK5D silica gel thin-layer chromatography (tlc) plates and neutral lipids separated using a one-dimensional, two-solvent system ( $n$-hexane:diethyl ether:acetic acid, 90:10:1, followed by the same components, 70:30:2) (Mangold, 1964). The following neutral lipid standards (Supelco, Inc.) were used : cholesterol; tripalmitin for triglycerides, palmitic acid for fatty acids, and cholesterol oleate for cholesterol esters. These compounds were located on tlc plates by spraying with phosphomolybdic acid in 5\% propanol (Pierce Chemical Co.). Neutral lipids in extracts, standards, and blanks, were measured spectrophotometrically after reaction of gels with acid dichromate (Amenta, 1964). Polar lipids were separated on the same tlc plates using chloroform:methanol:water $(65: 35: 3)$ as solvent (LePage, 1964). Lipid extract $(0.5-0.7 \mathrm{mg})$ was applied to the tlc plate. Phosphatidylcholine and phosphatidylethanolamine (Supelco, Inc.) were used as standards and were located by phosphomolybdic acid staining. Gels were eluted as described (Bienzenski, 1964) and phosphorus determined in $70 \%$ perchloric acid digests (Bartlett, 1959). Total phospholipid phosphorus was also measured following perchloric acid digestion of 0.1-0.3 mg lipid extract (Bartlett, 1959). Control experiments showed that extraction and recovery of all lipid classes analyzed exceeded $95 \%$.

Statistics. In experiments involving hypophysectomy and treatment with hormone, data were analyzed by the Student's $t$ test (one-sided).

\section{RESULTS}

Mean hepatic wet weight increased steadily from $83 \mathrm{mg}$ on 12 days of incubation to $1155 \mathrm{mg}$ on Day 21 . Liver dry weight increased from $15 \%$ of the wet weight on Day 12 to $36 \%$ on Day 21. The rate of increase in hepatic dry weight rose sharply after 16 days compared with a slower rise on Days 12-16. The accelerated accretion of lipid after 16 days is shown in Fig. 1. Also illustrated is the somewhat lower rate of increase in liver protein during this same period, and a steady decline in tissue hydration from 83.5\% water on Day 12 to $69.5 \%$ on Days 20 and 21 . In this report, liver contents of particular lipid classes are 


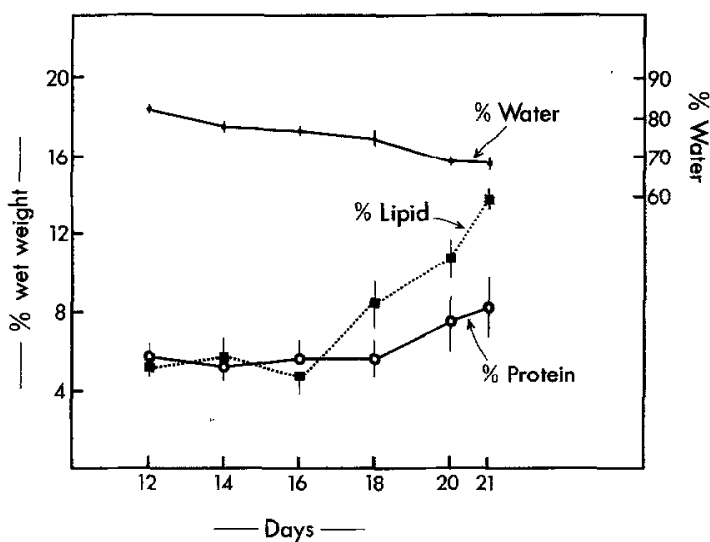

FIG. 1. Increased lipid and protein contents and decreased water content in embryonic chick liver from Day 12 to 21 of incubation.

normalized with respect to dry or to lipid weights rather than to wet weight. This is especially important in operated embryos as hypophysectomy elevated hepatic water content independently of its effects on liver biochemical composition (Fig. 2 below; cf. Sandra and Thommes, 1977).

Table 1 documents increased contents of certain hepatic lipid components, cholesterol, total phospholipid, and the major phospholipid, phosphatidylcholine, from Days 12 to 21 . When normalized to hepatic dry weight (which also increased during the same interval), the neutral lipid and phospholipids showed dissimilar ontogenetic patterns. Cholesterol content (per milligram of dry weight) increased from Days 12 to
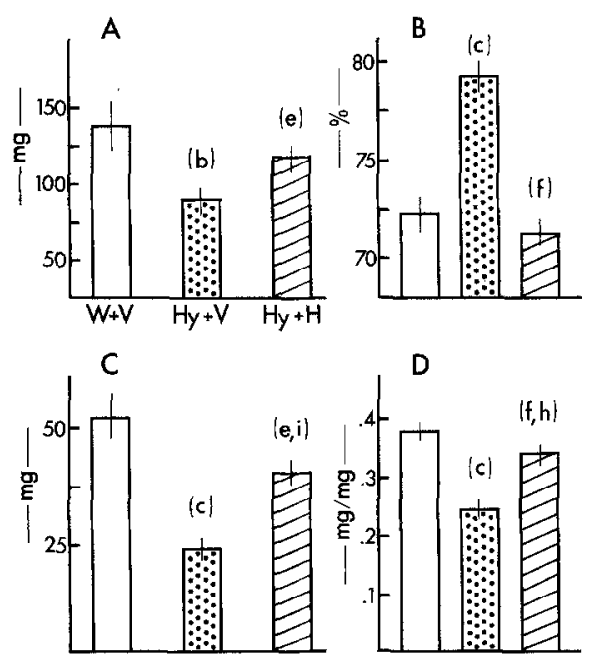

FIG. 2. Effects of hypophysectomy and 3 days treatment with vehicle or corticosterone acetate on liver composition in the 18-day embryo. (A) Liver dry weight (mg). (B) Hepatic percentage water. (C) Total liver lipid (mg). (D) Ratio of liver lipid content to liver dry weight (mg/mg). $N$ in each group: $\mathrm{W}+\mathrm{V}, 10 ; \mathrm{Hy}$ $+\mathrm{V}, 9 ; \mathrm{Hy}+\mathrm{H}, 8$. Other symbols defined in Table 2 .

18, but decreased on Day 20 and further at hatching. In contrast, fractional dry weight contributed by phospholipids declined between Days 12 and 14 but showed little further change to hatching. Fractional phosphatidylcholine content also varied little from Days 12 to 20 , but declined significantly $(P<0.05)$ at hatching (Table 1$)$.

Effects of hypophysectomy and corticosterone acetate treatment were observed on Days 16 and 18 of incubation. Results ob-

TABLE 1

Ontogeny of Liver Cholesterol, Phospholipid, and Phosphatidylcholine: Total Content and Content Normalized With Respect to Dry Weight

\begin{tabular}{|c|c|c|c|c|c|c|c|}
\hline \multirow{3}{*}{$\begin{array}{l}\text { Incubation } \\
\text { time } \\
\text { (days) }\end{array}$} & \multirow[b]{3}{*}{$N$} & \multicolumn{2}{|c|}{ Cholesterol } & \multirow{2}{*}{\multicolumn{2}{|c|}{ Phospholipid }} & \multirow{2}{*}{\multicolumn{2}{|c|}{ Phosphatidylcholine }} \\
\hline & & & $\mathrm{mg} / \mathrm{mg} \mathrm{dr}$ & & & & \\
\hline & & $\mathrm{mg}$ & $\left(\times 10^{3}\right)$ & $\mu \mathrm{mol}$ & $\mu \mathrm{mol} / \mathrm{mg}$ dry $\mathrm{wt}$ & $\mu \mathrm{mol}$ & $\mu \mathrm{mol} / \mathrm{mg}$ dry wt \\
\hline 12 & 5 & $0.12 \pm 0.02$ & $9.19 \pm 1.32$ & $2.02 \pm 0.15$ & $22.5 \pm 3.2$ & $0.83 \pm 0.15$ & $4.61 \pm 0.70$ \\
\hline 14 & 7 & $0.27 \pm 0.04$ & $8.69 \pm 0.64$ & $4.75 \pm 0.49$ & $12.2 \pm 1.0$ & $1.58 \pm 0.18$ & $5.18 \pm 0.71$ \\
\hline 16 & 7 & $0.62 \pm 0.12$ & $15.04 \pm 1.70$ & $6.46 \pm 0.88$ & $14.6 \pm 1.2$ & $1.93 \pm 0.17$ & $4.49 \pm 0.92$ \\
\hline 18 & 8 & $2.54 \pm 0.41$ & $27.01 \pm 2.08$ & $11.05 \pm 1.62$ & $11.8 \pm 0.9$ & $5.06 \pm 0.46$ & $5.99 \pm 0.35$ \\
\hline 20 & 5 & $4.41 \pm 0.77$ & $16.19 \pm 0.81$ & $12.87 \pm 0.95$ & $11.1 \pm 1.1$ & $8.91 \pm 0.72$ & $7.05 \pm 0.43$ \\
\hline 21 & 5 & $6.82 \pm 0.46$ & $14.45 \pm 1.88$ & $41.94 \pm 4.39$ & $10.8 \pm 2.1$ & $11.65 \pm 0.68$ & $2.63 \pm 0.73$ \\
\hline
\end{tabular}


tained using 16-day livers are summarized in Table 2. Hypophysectomized embryos showed minor (and statistically insignificant) reductions in total dry weight, lipid weight, and in cholesterol and phospholipid contents when compared with windowed controls. Hormone-treated hypophysectomized embryos displayed modestly increased hepatic dry weight, lipid weight, and cholesterol content, when compared with the hypophysectomized + vehicle group. Of these, only the elevation in total lipid weight was statistically significant $(P<0.05)$. Iivers of hypophysectomized hormone-treated embryos also displayed minor reductions in total phospholipid and phosphatidylcholine contents when compared with livers of hypophysectomized + vehicle-treated embryos.

In 18-day embryos, hypophysectomy produced statistically significant depressions in liver dry weight, total lipids, and in the ratio of lipid to dry weight when compared with windowed embryos (Fig. 2). Hormone treatment restored, or partially restored, each of these characteristics to near control values (Fig. 2). Hypophysec- tomy and glucocorticoid replacement had different effects on the hepatic contents of particular lipid components at 18 days. Cholesterol was significantly elevated above the hypophysectomized level by hormone treatment (Table 3). Liver phospholipid content, in contrast, was reduced by hypophysectomy, but was not restored by hormone. Instead, the two major phospholipid components, phosphatidylcholine and phosphatidylethanolamine; were further depressed by hormone treatment (Table 4).

Data in Table 5 summarize hepatic contents of cholesterol esters, triglycerides, and free fatty acids observed in two unoperated groups of embryos (18- and 21-day) and in 18-day control, hypophysectomized, and hormone-treated embryos. Liver cholesterol ester content was markedly reduced by hypophysectomy, but was not restored by hormone treatment. Hormonetreated hypophysectomized embryos, in fact, showed a further reduction in hepatic cholesterol esters below the comparatively low level produced by pituitary: ablation. Hepatic triglycerides were sharply in-

TABLE 2

EFfects of Hypophysectomy and TReatment with Vehicle or Corticosterone Acetate on Liver Lipid COMPOSITION aT 16 DaYs OF INCUBATION

\begin{tabular}{cccccc}
\hline Treatment & $N$ & $\begin{array}{c}\text { Lipid weight } \\
(\mathrm{mg})\end{array}$ & $\begin{array}{c}\text { Cholesterol } \\
(\mathrm{mg})\end{array}$ & $\begin{array}{c}\text { Phospholipid } \\
(\mu \mathrm{mol})\end{array}$ & $\begin{array}{c}\text { Phosphatidylcholine } \\
(\mu \text { mol })\end{array}$ \\
\hline W $+\mathrm{V}$ & 5 & $14.7 \pm 1.8$ & $0.73 \pm 0.10$ & $7.82 \pm 0.92$ & $3.02 \pm 0.33$ \\
Hy $+\mathrm{V}$ & 4 & $12.3 \pm 0.9$ & $0.51 \pm 0.08$ & $6.05 \pm 0.54$ & $4.17 \pm 0.47$ \\
Hy $+\mathrm{H}$ & 5 & $19.6 \pm 3.4^{*, t}$ & $0.66 \pm 0.07$ & $5.85 \pm 0.61$ & $3.68 \pm 0.43$ \\
\hline
\end{tabular}

Note. $\mathrm{W}+\mathrm{V}$, Windowed controls; $\mathrm{Hy}+\mathrm{V}$, hypophysectomized controls; $\mathrm{Hy}+\mathrm{H}$, hypophysectomized and hormone treated.

* Statistics: In Tables $2-6$ and in Fig. 2, statistical differences ( $t$ test, one-sided) are summarized according to the following code:

${ }^{a} \mathrm{Hy}+\mathrm{V}$ significantly different from $\mathrm{W}+\mathrm{V}$ (control), $P<0.05$.

${ }^{\circ} \mathrm{Hy}+\mathrm{V}$ significantly different from $\mathrm{W}+\mathrm{V}$ (control), $P<0.01$.

" $\mathrm{Hy}+\mathrm{V}$ significantly different from $\mathrm{W}+\mathrm{V}$ (control), $P<0.001$

" $\mathrm{Hy}+\mathrm{H}$ significantly different from $\mathrm{Hy}+\mathrm{V}$ (control), $P<0.05$.

' $\mathrm{Hy}+\mathrm{H}$ significantly different from $\mathrm{Hy}+\mathrm{V}$ (control), $P<0.01$.

$\mathrm{Hy}+\mathrm{H}$ significantly different from $\mathrm{Hy}+\mathrm{V}$ (control), $P<0.001$.

${ }^{g} \mathrm{Hy}+\mathrm{H}$ significantly different from $\mathrm{W}+\mathrm{V}$ (control), $P<0.05$.

${ }^{n} \mathrm{Hy}+\mathrm{H}$ significantly different from $\mathrm{W}+\mathrm{V}$ (control), $P<0.01$.

i Hy $+\mathrm{H}$ significantly different from $\mathrm{W}+\mathrm{V}$ (contral), $\boldsymbol{P}<\mathbf{0 . 0 0 1}$

Unlabeled values are not significantly different $(P<0.05)$ from controls. 
TABLE 3

EFFECTS OF Hypophysectomy AND TREATMENT with Vehicle or Corticosterone Acetate on Liver Cholesterol Content AT 18 DAYS OF INCUBATION

\begin{tabular}{crcc} 
& \multicolumn{2}{c}{ Cholesterol } \\
\cline { 3 - 4 } Treatment & $N$ & $\mathrm{mg}$ & $\mathrm{mg} / \mathrm{mg}$ lipid $\left(\times 10^{2}\right)$ \\
\hline $\mathrm{W}+\mathrm{V}$ & 10 & $3.37 \pm 0.38$ & $4.60 \pm 0.26$ \\
$\mathrm{Hy}+\mathrm{V}$ & 9 & $0.81 \pm 0.07^{c}$ & $3.46 \pm 0.38^{b}$ \\
$\mathrm{Hy}+\mathrm{H}$ & 8 & $1.76 \pm 0.18^{f, g}$ & $4.30 \pm 0.15^{e}$ \\
\hline
\end{tabular}

creased in hypophysectomized embryos compared with control and unoperated 18day embryos. Hepatic triglyceride concentration remained elevated in the corticosterone acetate-treated group. Most of the increase in lipid obtained in response to hormone could be attributed to gains in the triglycerides fraction. A modest (nonsignificant) rise in liver free fatty acids in hypophysectomized embryos was also not altered by hormone treatment.

\section{DISCUSSION}

\section{Ontogeny of Hepatic Lipids}

These data confirm that hepatic lipid content increases during ontogeny with the most marked rise occurring after 16 days of incubation (Fig. 1; Noble and Moore, 1964, 1967; Romanoff, 1960). In terms of particular lipid components, cholesterol and phospholipid (including phosphatidylcholine) content increased rapidly from 16 days on. When normalized to hepatic lipid content or dry weight, however, the proportion contributed by each component to the total liver lipid changed during on- togenesis in nonparallel fashion (Table 1). The period after 16 days of incubation corresponds with the incubation interval during which lipid transport from yolk to embryo is greatest (Noble and Moore, 1964) and also coincides with maturation of the pituitary-adrenal axis (Wise and Frye, 1973; Kalliecharan and Hall, 1974).

\section{Effects of Hypophysectomy on Hepatic Lipid Composition}

Most differences observed in livers of control and hypophysectomized 16-day embryos were minor (Table 2). The lack of clear differences in lipid content or composition in experimental and control groups may indicate that embryonic lipid metabolism or transport of fat from yolk functions without an important pituitary hormone requirement before 16 days. In contrast, hypophysectomy produced several changes in lipid composition of the liver of the 18-day chick embryo. Compared with intact-windowed controls, livers in the hypophysectomized group displayed reductions in dry weight, in total lipid, and in lipid per unit dry weight; hypophysectomy also increased hepatic water content (Fig. 2; Sandra and Thommes, 1977) and produced a decline in liver protein content (data not shown). Hypophysectomy reduced total liver cholesterol and also lowered its relative abundance in hepatic lipids (Table 3). This result seems consistent with the depressed serum cholesterol concentration observed in hypophysectomized embryos (Thommes and Shulman, 1967). Pituitary ablation also reduced total hepatic

TABLE 4

EfFects of Hypophysectomy and TreatMent With Vehicle or Corticosterone Acetate on Livfr Phosphoi.tpin Content and Composition at 18 Davs of Incubation

\begin{tabular}{|c|c|c|c|c|c|c|c|}
\hline \multirow[b]{2}{*}{ Treatment } & \multirow[b]{2}{*}{$N$} & \multicolumn{2}{|c|}{ Phospholipid ( $\mu \mathrm{mol})$} & \multicolumn{2}{|c|}{ Phosphatidylcholine ( $\mu \mathrm{mol})$} & \multicolumn{2}{|c|}{ Phosphatidylethanolamine ( $\mu \mathrm{mol})$} \\
\hline & & Total & mg lipid $^{-1}$ & Total & Ing lipid-1 & Total & mg lipid ${ }^{-1}$ \\
\hline $\mathbf{W}+\mathrm{V}$ & 10 & $14.35 \pm 1.90$ & $0.40 \pm 0.02$ & $7.34 \pm 0.36$ & $0.19 \pm 0.01$ & $2.13 \pm 0.25$ & $0.04 \pm 0.01$ \\
\hline $\mathrm{Hy}+\mathrm{V}$ & 9 & $9.95 \div 0.88^{c}$ & $0.39 \pm 0.03$ & $5.42 \pm 0.63^{c}$ & $0.27 \pm 0.03^{c}$ & $1.45 \pm 0.19^{c}$ & $0.08 \pm 0.01^{\circ}$ \\
\hline $\mathbf{H y}+\mathbf{H}$ & 8 & $9.77 \pm 0.76^{i}$ & $0.29 \pm 0.05^{e, h}$ & $4.61 \pm 0.52^{i}$ & $0.14 \pm 0.02^{f h}$ & $1.04 \pm 0.13^{c, i}$ & $0.03 \pm 0.01^{f}$ \\
\hline
\end{tabular}


TABLE 5

EFFects of Hypophysectomy and Treatment with Vehicle or Corticosterone Acetate on Hepatic Contents of Cholesterol Esters, Triglycerides, and

Free Fatty Acids at 18 Days of Incubation

\begin{tabular}{ccccc}
\hline & & \multicolumn{3}{c}{ mg/mg lipid $\left(\times 10^{2}\right)$} \\
\cline { 3 - 5 } Treatment & $N$ & Cholesterol esters & Triglycerides & Free fatty acids \\
\hline 21-Day intact & 4 & $45.06 \pm 3.81$ & $4.1 \pm 1.2$ & $0.49 \pm 0.15$ \\
18-Day intact & 4 & $37.18 \pm 2.51$ & $5.9 \pm 1.5$ & $0.49 \pm 0.13$ \\
18-Day W + V & 5 & $29.77 \pm 2.25$ & $9.1 \pm 1.8$ & $0.71 \pm 0.20$ \\
18-Day Hy + V & 5 & $9.19 \pm 0.83^{c}$ & $27.4 \pm 2.3^{c}$ & $1.17 \pm 0.32$ \\
18-Day Hy + H & 8 & $3.38 \pm 0.34^{e, i}$ & $32.1 \pm 2.7^{e, i}$ & $1.18 \pm 0.24$ \\
\hline
\end{tabular}

phospholipids, phosphatidylcholine, and phosphatidylethanolamine (Table 4). However, after normalization to lipid content the fractional contribution of phosphatidylcholine and phosphatidylethanolamine to total lipid actually increased. That is, the decrease in these two phospholipid components was relatively less than the decline in lipid content generally. Therefore, one effect of hypophysectomy was to selectively alter the proportions of particular lipid classes.

Cholesterol esters are quantitatively the most important lipid class in the avian embryonic liver, accounting for about $30 \%$ of the dry weight in the 19-day embryo (Moore and Doran, 1962; Noble and Moore, 1964). Cholesterol ester content was especially sensitive to hypophysectomy. Hepatic lipid in the hypophysectomized group possessed only $30 \%$ of the esterified cholesterol contained in windowed controls, but displayed increased hepatic triglyceride content (Table 5). Increased triglyceride and decreased cholesterol ester concentrations have also been observed in the livers of vitamin $B_{12}$-deficient embryos (Noble and Moore, 1964) and may signal severe metabolic disturbance.

\section{Effects of Treatment with \\ Corticosterone Acetate}

At issue is whether liver lipid content or composition is controlled by adrenal glucocorticoids. Treatment with corticoste- rone acetate $(300 \mu \mathrm{g} /$ day on Days 13,14 , and 15) elevated liver lipid content above that seen in hypophysectomized livers in 16- and 18-day embryos (Table 2, Fig. 2). Hormone treatment also raised liver protein content (data not shown) and reduced the tissue hydration typically observed after hypophysectomy (Fig. 2). Alterations in the liver content of particular types of lipids in response to hormonal treatment was highly lipid specific. Cholesterol content in 18-day livers was elevated by glucocorticoid to twice the hypophysectomized level, but did not reach the higher content observed in the intact group (Table 3). In contrast, total hepatic phospholipid and its two major components, phosphatidylcholine and phosphatidylethanolamine, were not increased above the depressed hypophysectomized value in response to hormone. Glucocorticoid treatment actually caused a further decline in the relative proportions of the major hepatic phospholipids (Table 4). Corticosterone acetate treatment neither restored the loss of hepatic cholesterol esters observed after hypophysectomy, nior did this hormone lower elevated liver trglycerides and free fatty acids measured in hypophysectomized embryos (Table 5). An important conclusion can be drawn with respect to the types of hepatic lipids lost (after hypophysectomy) and gained (after corticosterone treatment of hypophysectomized embryos). The major constituents lost were cholesterol esters, whereas the 
greatest gain in response to hormone was in triglycerides.

The experiments reported in this paper demonstrate an important role for pituitary hormones in the regulation of hepatic lipid content in the chick embryo. One or more pituitary hormones are required for normal development of liver lipid composition, and this requirement is most stringent after 16 days of incubation. The failure of corticosterone acetate to elevate individual and total phospholipids and cholesterol esters, as well as its inability to lower triglyceride concentration to levels seen in intact embryos, argues that control of these important liver lipids may depend on other hormones which are also reduced or eliminated by hypophysectomy. One possible agent in thyroid hormone, the secretion of which is controlled by pituitary TSH in the chick embryo from Day 12.5 (Thommes et al., 1977). $T_{3}$ is lipogenic in hepalocytes isolated from the 19-day chick and acts with insulin to increase acetyl-CoA carboxylase, fatty acid synthetase (Fisher and Goodridge, 1978), and malic enzyme (Goodridge, 1978; Joshi and Aranda, 1979b). With respect to yolk sac development and function per se, $T_{3}$ and $T_{4}$ appear to promote retraction of this membrane during the period of maximal fat absorption on Days 19-20 (Wishart et al., 1977). Further investigations are required to establish the roles of thyroid hormone and also of insulin in embryonic lipid metabolism and transport.

Sites of pituitary and adrenal hormone actions cannot be located from the results of these studies. As the metabolic consequences of hypophysectomy are widespread and severe (Thommes and Shulman, 1967), many metabolic pathways which are directly or only indirectly concerned with lipid metabolism or transport will also be disturbed by partial decapitation. However, the notable decline of hepatic cholesterol esters and phospholipids after hypophysectomy suggest direct or indirect effects of pituitary hormones on yolk absorption. Both kinds of lipids originate in the yolk and are modified by the yolk sac membrane during absorption (Noble and Moore, 1964, 1967). Cholesterol esters are especially important for the assembly of lipoproteins which move lipid from yolk to embryo (Freeman, 1978). These esters appear to be synthesized by blood and yolk sac lecithin-cholesterol acyl transferases which use cholesterol transported from yolk by very low density lipoproteins as substrate (Noble and Moore, 1967; Bengtsson et al., 1977). Cholesterol ester so formed are then deposited and stored in the embryonic liver. Hypophysectomy interferes at some point with this transport sequence which normally translocates yolk lipids to the liver.

\section{ACKNOWLEDGMENTS}

The author is grateful to V. Hylka for technical advice. Dr. R. Marsh reviewed an earlier version of this paper. The manuscript was typed by Ms. S. Schaerer; illustrations were drawn by Ms. P. Wander. Supported by a Rackham Faculty grant.

\section{REFERENCES}

Amenta, J. S. (1964). A rapid chemical method for quantification of lipids by thin-layer chromatography. J. Lipid Res. 5, 270-272.

Bartlett, G. R. (1959). Phosphorus assay in column chromatography. J. Biol. Chem. 234, 466-468.

Bengtsson, G., Hernell, O., and Olivecrona, T. (1977). Are egg yolk lipoproteins metabolized by the chick embryo in the same manner as plasma lipoproteins are in the adult? Int. J. Biochem. 8 , $587-589$.

Bienzenski, J. J. (1964). Quantitation and preparation of phospholipids by elution following improved thin layer chromatography separation. Fed. Proc. 23, 503 .

Donaldson, W. E., Mueller, N. S., and Mason, J. V. (1971). Intracellular localization of fatty acid synthesis in chick embryo liver and stimulation of synthesis by exogenous glucose. Biochim. Biophys. Acta 248, 34-40.

Fisher, P. W. F., and Goodridge, A. G. (1978). Coordinate regulation of acetyl Coenzyme A carboxylase and fatty acid synthetase in liver cells of the developing chick in vivo and in culture. Arch. Biochem. Biophys. 190, 332-344. 
Folch, J., Lees, M., and Stanley, G. H. S. (1957). A simple method for the isolation and purification of total lipides from animal tissues. J. Biol. Chem. 226, 497-509.

Freeman, B. M. (1978). Chemical embryology. In "Chemical Zoology," Vol. 10, "Aves" (A. H. Bursh, ed.), pp. 75-115. Academic Press, New York.

Fugo, N. W. (1940). Effects of hypophysectomy in the chick embryo. J. Exp. Zool. 85, 271-297.

Goodridge, A. G. (1968). Conversion of $\left[\mathrm{U}-\mathrm{C}^{14}\right]$ glucose into carbon dioxide, glycogen, cholesterol and fatty acids in liver slices from embryonic and growing chicks. Biochem. J. 108, 655-661.

Goodridge, A. G. (1968b). Metabolism of glucose-U${ }^{14} \mathrm{C}$-in vitro in adipose tissue from embryonic and growing chicks. Amer. J. Physiol. 214, 897-901.

Goodridge, A. G. (1968c). Citrate-cleavage enzyme, 'malic' enzyme and certain dehydrogenases in embryonic and growing chicks. Biochem. J. 108, $663-666$.

Goodridge, A. G. (1978). Regulation of malic enzyme synthesis by thyroid hormone and glucagon: Inhibitor and kinetic experiments. Mol. Cell. Endocrinol. 11, 19-29.

Goodridge, A. G., and Ball, E. G. (1967). The effect of prolactin on lipogenesis in the pigeon. In vivo studies. Biochemistry 6, 1676-1681.

Joshi, V. C., and Aranda, L. P. (1979a). Hormonal regulation of the terminal enzyme of microsomal stearoyl coenzyme A desaturase in cultured avian liver explants. Role of insulin. J. Biol. Chem. 254, $1179-1182$.

Joshi, V. C., and Aranda, L. P. (1979b). Hormonal regulation of fatty acid synthetase in cultured avian liver explants. Role of insulin. J. Biol. Chem. 254, 11783-11786.

Joshi, V. C., and Sidbury, J. B. (1975). Fatty acid synthesis in chick embryonic heart and liver during development. Develop. Biol. 42, 282-291.

Kalliecharan, R., and Hall, B. K. (1974). A developmental study of the levels of progesterone, corticosterone, cortisol, and cortisone circulating in plasma of chick embryos. Gen. Comp. Endocrinol. 24, 364-372.

Langslow, D. R. (1972). The development of lipolytic sensitivity in the isolated fat cells of Gallus domesticus during the foetal and neonatal period. Comp. Biochem. Physiol. 43B, 689-701.
Langslow, D. R., Cramb, G., and Siddle, K. (1979). Possible mechanisms for the increased sensitivity to glucagon and catecholamines of chicken adipose tissue during hatching. Gen. Comp. Endocrinol. 39, 527-533.

LePage, M. (1964). Isolation and characterization of an esterified form of a steryl glucoside. $J$. Lipid Res. 5, 587-592.

Lowry, O. H., Rosebrough, N. J., Farr, A. L., and Randall, R. J. (1951). Protein measurement with the Folin phenol reagent. J. Biol. Chem. 193, 264-275.

Mangold, H. K. (1964). Thin layer chromatography of lipids. J. Amer. Oil Chem. Soc. 38, 708-727.

Moore, J. H., and Doran, B. M. (1962). Lipid metabolism in the normal and vitamin $B_{:^{2}}$ deficient chick embryo. Biochem. $J .84,506-513$.

Noble, R. C., and Moore, J. H. (1964). Studies on the lipid metabolism of the chick embryo. Canad. $J$. Biochem. 42, 1729 -1741.

Noble, R. C., and Moore, J. H. (1967). The liver phospholipids of the developing chick embryo. Canad. J. Biochem. 45, 627-639.

Pugh, E., and Sidbury, J. B. (1971). Fatty acid oxidation in embryonic chick tissues. Biochim. Biophys. Acta 239, 376-383.

Romanoff, A. L. (1960). "The Avian Embryo," pp. 521-524. Macmillan Co., New York.

Sandra, A., and Thommes, R. C. (1977). Modulation of lipids in the chick liver by "hypophysectomy" and replacement. Growth 41, 199-206.

Thommes, R. C., and Shuman, R: W. (1967). Endocrine control of lipid metabolism in the developing chick embryo. I. Blood cholesterol. Gen. Comp. Endocrinol. 8, 54-60.

Thommes, R. C., Vieth, R. L., and Levasseur, S. (1977). The effects of hypophysectomy by means of surgical decapitation on thyroid function in the developing chick embryo. I. Plasma thyroxine. Gen. Comp. Endocrinol. 31, 29-36.

Wise, P., and Frye, B. E. (1973). Functional development of the hypothalamohypophyseal-adrenal cortex axis in the chicken embryo (Gallus dorresticus). J. Exp. Zool. 185, 277-291.

Wishart, G. J., Leakey, J. E. A., and Dutton, G. J. (1977). Differential effects of hormones on precocious yolk sac retraction in chick embryos following administration by a new technique. Ger. Comp. Endocrinol. 31, 373-380. 